\title{
Some rigorous results on the phase transition of finitary random interlacements*
}

\author{
Zhenhao Cai $^{\dagger} \quad$ Yuan Zhang $^{\ddagger}$
}

\begin{abstract}
In this paper, we show several rigorous results on the phase transition of Finitary Random Interlacements (FRI). For the high intensity regime, we show the existence of a critical fiber length, and find its exact asymptotic as intensity goes to infinity. At the same time, our result for the low intensity regime proves the global existence of a non-trivial phase transition with respect to the system intensity.
\end{abstract}

Keywords: finitary interlacements; percolation; phase transition; critical value. MSC2020 subject classifications: $60 \mathrm{~K} 35$.

Submitted to ECP on February 5, 2021, final version accepted on August 29, 2021.

\section{Introduction}

The model of finitary random interlacements (FRI) was first introduced by Bowen [1] to solve a special case of the Gaboriau-Lyons problem. Intuitively speaking, FRI (denoted by $\mathcal{F I}^{u, T}$ ) can be seen as a "cloud of twisted yarn" composed of finite "fibers" on $\mathbb{Z}^{d}$. The fibers within this system each forms a geometrically killed simple random walk with an expected length $T$. Meanwhile, the starting point of which is sampled according to a Poisson point process with intensity measure proportional to system intensity $u$ and inversely proportional to $T+1[1,2,3,8]$. See Section 2 for precise definitions.

In [1], Bowen proved that as $T \rightarrow+\infty, \mathcal{F I}^{u, T}$ converges under weak $*-$ topology to the celebrated random interlacements (RI) $\mathcal{I}^{u}$ introduced by Sznitman [10]. At the same time, FRI can also be seen as a finitary version of RI, as it can be defined as massive interlacements on a graph equipped with killing measure (see Chapter V of [7]).

On the other hand, unlike the classic system of random interlacements, whose trajectories always form an a.s. connected network [4, 10], the collection of edges traversed by FRI on $\mathbb{Z}^{d}, d \geq 3$ has exhibited a non-trivial percolative phase transition [8]. And since there are now two parameters to play with, one may characterize such transition on phase space $\{(u, T):, u>0, T>0\}$ from either of the following aspects:

(1) One may first fix the intensity $u$ and examine the evolution of FRI with respect to $T$. This is also the problem Bowen studied in his first paper [1]. In addition to the local convergence as $T \rightarrow \infty$, Bowen also proved that the FRI on non-amenable graphs will a.s. have infinite connected cluster(s) for all sufficiently large $T$. He proposed a

\footnotetext{
*Research supported by National Key R\&D Program of China, Project Number 2020YFA0712902.

${ }^{\dagger}$ Peking University, China. E-mail: caizhenhao@pku.edu.cn

${ }^{\ddagger}$ Peking University, China.

E-mail: zhangyuan@math.pku.edu.cn https://www.math.pku.edu.cn/teachers/zhangyuan/eindex.html
} 
Rigorous results on the phase transition of FRI

parallel question for FRI on $\mathbb{Z}^{d}$, which was affirmatively answered in [8], where the existence of the following phase transition with respect to $T$ was proved: For any $d \geq 3$ and $u>0$, there are $T_{0}, T_{1} \in(0, \infty)$, such that $\mathcal{F I}^{u, T}$ a.s. has no infinite cluster for all $T<T_{0}$, and a.s. has a unique infinite cluster for all $T>T_{1}$ (see Theorem 1 , 2 in [8] for details). The geometric properties of this infinite cluster, such as local uniqueness and order of chemical distance, for sufficiently large $T$, was later obtained in [2]. However, as mentioned in Section 1.1 of [8], the uniqueness of phase transition and the existence/uniqueness of a critical fiber length $T_{c}$ remain open. Unlike the case of RI, this turns out to be a highly non-trivial problem, since it was recently proved that there is no global stochastic monotonicity with respect to $T$ for $\mathcal{F} \mathcal{I}^{u, T}$. See Theorem 1, [3] for details. On the other hand, numerical simulations in Section 5 of [3] provided evidences on the existence and uniqueness of $T_{c}$. It was further conjectured in Conjecture 5 [3] that $T_{c}$ is asymptotically an inverse linear function with respect to $u$.

(2) As in [7], one may also fix $T$ and examine the evolution of FRI with respect to its intensity $u$. Note that FRI is by definition monotone with respect to $u$. So the question of interest here is whether there is always a non-trivial phase transition. I.e., for a fixed $T$, we want to show FRI does not percolate for all sufficiently small $u$ and percolate for all sufficiently large $u$. [7] proved this for all $T$ small enough, and conjectured it can be extended to all $T \in(0, \infty)$.

In this paper, we prove that, despite lacking global monotonicity, FRI is stochastically increasing with respect to $T$ for all $T \in(0,1)$, which implies the existence and uniqueness of $T_{c}$ for all sufficiently large $u$. Meanwhile, we also show that the upper bound of $T_{c}$ found by $[3,7]$ in the high intensity regime is actually sharp in the limit, and give an exact asymptotic of $T_{c}$ as $u \rightarrow \infty$. Moreover, for the low intensity regime, we prove a polynomial lower bound for the phase diagram, which at the same time proves the conjecture on the global existence of a non-trivial phase transition with respect to $u$. Our proofs are largely based on the "decoupling" methods first invented in [11].

This paper is organized as follows: In Section 2 we introduce precise definitions of FRI together with necessary notations and preliminaries. We state our main results in Section 3. Local monotonicity is shown in Section 4. And at last we estimate the asymptotic of critical values in Section 5.

\section{Notations and preliminaries}

Some basic notations: In this paper, we denote the $l^{\infty}$ distance and Euclidean distance by $|\cdot|$ and $|\cdot|_{2}$ respectively. The undirected edge set of $\mathbb{Z}^{d}$ is denoted by $\mathbb{L}^{d}$ (i.e. $\mathbb{L}^{d}:=\left\{\{x, y\}: x, y \in \mathbb{Z}^{d},|x-y|_{2}=1\right\}$ ). For any subsets $A, B \subset \mathbb{Z}^{d}$, the $l^{\infty}$ distance between them is defined as $d(A, B):=\min \{|x-y|: x \in A, y \in B\}$. For finite subset $D \subset \mathbb{Z}^{d}$, let $\partial D:=\left\{x \in D: \exists y \in \mathbb{Z}^{d} \backslash D\right.$ such that $\left.\{x, y\} \in \mathbb{L}^{d}\right\}$ be its inner boundary and $|D|$ be the cardinality of $D$, without causing further confusion.

Connection between two sets: For sets $A, B \subset \mathbb{Z}^{d}$ and a collection of edges in $\mathbb{L}^{d}$ denoted by $E$, we say $A$ and $B$ are connected by $E$ (written by $A \stackrel{E}{\leftrightarrow} B$ ) iff there exists a sequence of vertices $\left(x_{0}, \ldots, x_{n}\right)$ such that $x_{0} \in A, x_{n} \in B$ and that for any $0 \leq i \leq n-1$, $\left\{x_{i}, x_{i+1}\right\} \in E$.

Statements on constants: we will use $c, c_{1}, c_{2}, \ldots$ as local constants ("local" means their values may vary according to contexts) and $C, C_{1}, C_{2}, \ldots$ as global constants ("global" means constants will keep their values throughout the whole paper).

Random walks and relative stopping times: We denote the law of simple random walks starting from $x$ on $\mathbb{Z}^{d}$ by $P_{x}$ and the law of geometrically killed simple random walks starting from $x$ with killing rate $\frac{1}{T+1}$ at each step by $P_{x}^{(T)}$.

For a random walk $\left\{X_{i}\right\}_{n=0}^{\infty}$ and $A \subset \mathbb{Z}^{d}$, define the corresponding hitting time and 
entrance time as $H_{A}=\min \left\{k \geq 0: X_{k} \in A\right\}$ and $\bar{H}_{A}=\min \left\{k \geq 1: X_{k} \in A\right\}$. Here we use the convention that $\min \emptyset=\infty$.

Capacity with killing measure: For any $K \subset \mathbb{Z}^{d}$, the escaping probability is defined as $E s_{K}^{(T)}(x):=P_{x}^{(T)}\left(\bar{H}_{K}=\infty\right)$. Then the capacity of $K$ with killing measure $\frac{1}{T+1}$ is defined by

$$
\operatorname{cap}^{(T)}(K):=2 d * \sum_{x \in K} E s_{K}^{(T)}(x) .
$$

In particular, we note the following trivial upper bound

$$
\operatorname{cap}^{(T)}(K) \leq 2 d *|K|
$$

Definitions of FRI: According to [8], FRI has two equivalent definitions. Denote the set of all finite nearest-neighbor paths on $\mathbb{Z}^{d}$ by $W^{[0, \infty)}$. Then $v^{(T)}:=\sum_{x \in \mathbb{Z}^{d}} \frac{2 d}{T+1} P_{x}^{(T)}$ is a $\sigma$-finite measure on $W^{[0, \infty)}$.

Definition 2.1. For $0<u, T<\infty$, finitary random interlacements $\mathcal{F I}^{u, T}$ is the Poisson point process with intensity measure $u * v^{(T)}$. We denote the law of $\mathcal{F} \mathcal{I}^{u, T}$ by $P^{u, T}$.

Definition 2.2. Let $\left\{N_{x}\right\}_{x \in \mathbb{Z}^{d}} \stackrel{\text { i.i.d. }}{\sim}$ Pois $\left(\frac{2 d u}{T+1}\right)$. For each site $x \in \mathbb{Z}^{d}$, start $N_{x}$ independent geometrically killed simple random walks with law $P_{x}^{(T)}$. Let $\mathcal{F I}^{u, T}$ be the point measure on $W^{[0, \infty)}$ composed of all the trajectories above starting from all $x \in \mathbb{Z}^{d}$.

With a slight abuse of notations, for $\eta \in W^{[0, \infty)}$, we may write $\eta \in \mathcal{F I}^{u, T}$ if $\mathcal{F} \mathcal{I}^{u, T}(\eta)=$ 1. In this paper, $\mathcal{F} \mathcal{I}^{u, T}$ is also regarded as a bond percolation model on $\mathbb{L}^{d}$. I.e. we say an edge $e \in \mathbb{L}^{d}$ is open in the FRI iff there exists a path in $\mathcal{F I}^{u, T}$ containing $e$, denoted by $\mathcal{F} \mathcal{I}^{u, T}(e)=1$ (otherwise, $\mathcal{F} \mathcal{I}^{u, T}(e)=0$ ). For the simplicity of notations, for any $A, B \subset \mathbb{Z}^{d}$, we denote

$$
A \stackrel{\mathcal{F I}^{u, T}}{\longrightarrow} B:=A \stackrel{\left\{e \in \mathbb{L}^{d}: \mathcal{F I}^{u, T}(e)=1\right\}}{\longrightarrow} B .
$$

We say $\mathcal{F} \mathcal{I}^{u, T}$ percolates iff there exists an infinite connected cluster composed of open edges. It has been proved in Theorem 2, [3] that $\mathcal{F} \mathcal{I}^{u, T}$ contains at most one open infinite connected cluster.

FRI on a finite set: Let $K$ be a finite subset of $\mathbb{Z}^{d}$. For each path $\eta_{i}$ in $\mathcal{F \mathcal { I } ^ { u , T }}$, we denote the part of $\eta_{i}$ after intersecting $K$ by $\hat{\eta}_{i}^{K}$. Precisely, for path $\eta_{i}=\left(\eta_{i}(0), \ldots, \eta_{i}\left(l_{i}\right)\right)$, define $\hat{\eta}_{i}^{K}:=\left(\eta_{i}\left(H_{K}\right), \ldots, \eta_{i}\left(l_{i}\right)\right)$ if $H_{K}<\infty$ and $\hat{\eta}_{i}^{K}:=\emptyset$ if $H_{K}=\infty$. By Lemma 2.1 of [8], $\sum_{i} \delta_{\hat{\eta}_{i}^{K}}$ has the same law as a Poisson point process with intensity measure $u * \sum_{x \in K} E s_{K}^{(T)}(x) P_{x}^{(T)}$. As a direct corollary, the number of paths intersecting $K$ in $\mathcal{F I}^{u, T}$ is a Poisson random variable with parameter $u * c a p^{(T)}(K)$.

Independence in FRI: For disjoint sets $A_{1}, A_{2}, \ldots, A_{m} \subset \mathbb{Z}^{d}$, consider an arbitrary sequence of events $E_{1}, E_{2}, \ldots, E_{m}$ satisfying that for all $1 \leq i \leq m, E_{i}$ depends only on the paths in $\mathcal{F I}^{u, T}$ starting from $A_{i}$. By Definition $2.2, E_{1}, E_{2}, \ldots, E_{m}$ are mutually independent.

Critical values of FRI:

Definition 2.3. For $u>0, d \geq 3$, we define that

$$
T_{c}^{-}(u, d):=\sup \left\{T_{0}>0: \forall 0<T<T_{0}, \mathcal{F I}^{u, T} \text { does not percolate }\right\}
$$

and

$$
T_{c}^{+}(u, d):=\inf \left\{T_{0}>0: \forall T>T_{0}, \mathcal{F I}^{u, T} \text { percolates }\right\} .
$$

Remark 2.4. By Theorem 1 and 2 of [8], it has been proved that for all $d \geq 3$ and $u>0$, $0<T_{c}^{-}(u, d) \leq T_{c}^{+}(u, d)<\infty$. 


\section{Main results}

In this paper, we first show that for all $d \geq 3$ and $u>0, \mathcal{F} \mathcal{I}^{u, T}$ is stochastically increasing with respect to $T$ for all $T \in(0,1]$. It is worth noting that $\mathcal{F I}^{u, T}$ has been proved not enjoying monotonicity for larger $T$ 's (see Theorem 1, [3]).

Theorem 3.1. For any $u>0,0<T_{1}<T_{2}<\infty$ such that $T_{1} * T_{2} \leq 1$, then $\mathcal{F I}^{u, T_{2}}$ stochasitcally dominates $\mathcal{F I}^{u, T_{1}}$. I.e. there is a coupling between $\mathcal{F I}^{u, T_{1}}$ and $\mathcal{F I}^{u, T_{2}}$ such that almost surely for any edge $e \in \mathbb{L}^{d}, \mathcal{F I}^{u, T_{1}}(e) \leq \mathcal{F} \mathcal{I}^{u, T_{2}}(e)$.

By Theorem 3.1, we know that for any $d \geq 3$ and $u>0$, if $0<T_{c}^{-} \leq T_{c}^{+}<1$, then there must exist a unique critical fiber length between 0 and 1 . In fact, according to the upper bound of $T_{c}^{+}$and the lower bound of $T_{c}^{-}$given in Theorem 3 (iv) and Proposition 2, [3], $0<T_{c}^{-} \leq T_{c}^{+}<1$ holds for all sufficiently large $u$. Therefore, we have the following corollary:

Corollary 3.2. For all $d \geq 3$, there is a $U_{d}<\infty$ such that for all $u \geq U_{d}$,

$$
T_{c}^{+}(u, d)=T_{c}^{-}(u, d):=T_{c}(u, d) .
$$

I.e., $\mathcal{F I}^{u, T}$ percolates a.s. for all $T>T_{c}$, and does not percolates a.s. for all $T<T_{c}$.

Our next result provides the exact asymptotic of $T_{c}$ as $u \rightarrow \infty$, which gives an affirmative answer to Part 2, Conjecture 5, [3].

Theorem 3.3. For all $d \geq 3$,

$$
\lim _{u \rightarrow \infty} u * T_{c}(u, d)=\frac{-\log \left(1-p_{d}^{c}\right)}{2},
$$

where $p_{d}^{c}$ is the critical value of Bernoulli bond percolation on $\mathbb{L}^{d}$.

Remark 3.4. In Theorem 4.2 [3] and on Page 263 [7], it has been shown that

$$
\limsup _{u \rightarrow \infty} u * T_{c}^{+} \leq \frac{-\log \left(1-p_{d}^{c}\right)}{2} .
$$

So here we only need to prove an asymptotically sharp lower bound for the subcritical phase (a partial result on the asymptotic order was given in Proposition 2, [3]).

For the low intensity regime (i.e. $u \ll 1$ ), we obtain the following estimate on the subcritical phase:

Theorem 3.5. For any $d \geq 3$ and $\delta>0$, there exist constants $0<U_{0}(d, \delta)<1$ and $C_{1}(d, \delta)>0$ such that for any $0<u \leq U_{0}$,

$$
T_{c}^{-} \geq C_{1} u^{-\frac{1}{d-1}+\delta} .
$$

Combining Theorem 3.5 and the supercritical estimates obtained in (v) of Theorem 3, [3],

Corollary 3.6. When $d=3$,

$$
0.5 \leq \liminf _{u \rightarrow 0} \frac{\log \left(T_{c}^{-}\right)}{-\log (u)} \leq \limsup _{u \rightarrow 0} \frac{\log \left(T_{c}^{+}\right)}{-\log (u)} \leq 2 ;
$$

When $d \geq 4$,

$$
\frac{1}{d-1} \leq \liminf _{u \rightarrow 0} \frac{\log \left(T_{c}^{-}\right)}{-\log (u)} \leq \limsup _{u \rightarrow 0} \frac{\log \left(T_{c}^{+}\right)}{-\log (u)} \leq 1 .
$$

At the same time, Theorem 3.3 together with Theorem 3.5 also give an affirmative answer to the conjecture proposed on Page 263, [7] which predicted the global existence of a non-trivial phase transition with respect to $u$ :

Theorem 3.7. For all $d \geq 3$ and $T \in(0, \infty)$, there is a $u_{c}=u_{c}(d, T) \in(0, \infty)$ such that $\mathcal{F I}^{u, T}$ percolates a.s. for all $u \in\left(u_{c}, \infty\right)$, and does not percolate a.s. for all $u \in\left(0, u_{c}\right)$. 


\section{Local stochastic monotoncity}

In order to prove Theorem 3.1, we introduce a new mechanism to construct $\mathcal{F} \mathcal{I}^{u, T}$. Denote the collection of all directed nearest-neighbor edges by $\hat{\mathbb{L}}^{d}:=\left\{x \rightarrow y:\{x, y\} \in \mathbb{L}^{d}\right\}$. Let $\left\{N_{x \rightarrow y}\right\}_{x \rightarrow y \in \hat{\mathbb{L}}^{d}} \stackrel{\text { i.i.d. }}{\sim}$ Pois $\left(\frac{u T}{(T+1)^{2}}\right),\left\{Y_{k}\right\}_{k=1}^{\infty} \stackrel{\text { i.i.d. }}{\sim} \operatorname{Geo}\left(\frac{T}{T+1}\right)$ and $\left\{X^{y, k}\right\}_{k \in \mathbb{N}^{+}, y \in \mathbb{Z}^{d}}$ be a sequence of independent random walks with law $P_{0}^{(T)}$. For $u \geq 0$, we have the following equivalence in distribution:

\section{Lemma 4.1.}

$$
\bigcup_{x \rightarrow y \in \hat{\mathbb{L}}^{d}} \bigcup_{1 \leq k \leq N_{x \rightarrow y}, Y_{k} \geq 1}\left\{\{x, y\},\left\{X_{i}^{y, k}, X_{i+1}^{y, k}\right\}, 0 \leq i \leq Y_{k}-1\right\} \stackrel{d}{=} \mathcal{F} \mathcal{I}^{u, T} .
$$

Proof. For any $x \in \mathbb{Z}^{d}$ and $x \rightarrow y \in \hat{\mathbb{L}}^{d}$, by Definition 2.2 and property of Poisson point process, we note that the number of paths starting from $x$ with length $\geq 1$ and the first step $x \rightarrow y$ is a Poisson variable with parameter $\frac{T}{T+1} * \frac{1}{2 d} * \frac{2 d u}{T+1}=\frac{u T}{(T+1)^{2}}$. In addition, by the memoryless property of geometric distribution, the remaining part of each path after the first step removed is still a geometrically killed random walk with killing rate $\frac{1}{T+1}$. Then Lemma 4.1 follows.

Now Theorem 3.1 can be proved as follows:

Proof of Theorem 3.1: When $T_{1}<T_{2}, 0<T_{1} * T_{2}<1$, we define three independent sequences of random variables:

$$
\begin{aligned}
& \left\{N_{x \rightarrow y}^{(1)}\right\} \stackrel{i . i . d .}{\sim} \text { Pois }\left(\frac{u T_{1}}{\left(T_{1}+1\right)\left(T_{2}+1\right)}\right), \\
& \left\{N_{x \rightarrow y}^{(2)}\right\} \stackrel{\text { i.i.d. }}{\sim} \text { Pois }\left(\frac{u T_{1}\left(T_{2}-T_{1}\right)}{\left(T_{1}+1\right)^{2}\left(T_{2}+1\right)}\right)
\end{aligned}
$$

and

$$
\left\{N_{x \rightarrow y}^{(3)}\right\} \stackrel{\text { i.i.d. }}{\sim} \text { Pois }\left(\frac{u\left(T_{2}-T_{1}\right)\left(1-T_{1} T_{2}\right)}{\left(T_{1}+1\right)^{2}\left(T_{2}+1\right)^{2}}\right) .
$$

Meanwhile, one may construct

$$
\left\{\left(Y_{k}^{\left(T_{1}\right)}, Y_{k}^{\left(T_{2}\right)}\right)\right\}_{k=1}^{\infty} \stackrel{i . i . d .}{\sim}\left(\operatorname{Geo}\left(\frac{T_{1}}{T_{1}+1}\right), \operatorname{Geo}\left(\frac{T_{2}}{T_{2}+1}\right)\right)
$$

such that for any $k \geq 1, P\left(Y_{k}^{\left(T_{1}\right)} \leq Y_{k}^{\left(T_{2}\right)}\right)=1$.

Note that for any $x \rightarrow y, N_{x \rightarrow y}^{(1)}+N_{x \rightarrow y}^{(2)} \sim$ Pois $\left(\frac{u T_{1}}{\left(T_{1}+1\right)^{2}}\right)$ and $N_{x \rightarrow y}^{(1)}+N_{x \rightarrow y}^{(2)}+N_{x \rightarrow y}^{(3)} \sim$ Pois $\left(\frac{u T_{2}}{\left(T_{2}+1\right)^{2}}\right)$. By Lemma 4.1, we have

$$
\bigcup_{x \rightarrow y \in \hat{\mathrm{L}}^{d}} \bigcup_{1 \leq k \leq N_{x \rightarrow y}^{(1)}+N_{x \rightarrow y}^{(2)}, Y_{k}^{\left(T_{1}\right)} \geq 1}\left\{\{x, y\},\left\{X_{i}^{y, k}, X_{i+1}^{y, k}\right\}, 0 \leq i \leq Y_{k}^{\left(T_{1}\right)}-1\right\} \stackrel{d}{=} \mathcal{F} \mathcal{I}^{u, T_{1}}
$$

Note that for any $k \geq 1$, if $Y_{k}^{\left(T_{1}\right)} \geq 1$, then $Y_{k}^{\left(T_{2}\right)} \geq 1$. Use Lemma 4.1 again,

$$
\bigcup_{x \rightarrow y \in \hat{\mathbf{L}}^{d}} \bigcup_{1 \leq k \leq N_{x \rightarrow y}^{(1)}+N_{x \rightarrow y}^{(2)}+N_{x \rightarrow y}^{(3)}, Y_{k}^{\left(T_{2}\right)} \geq 1}\left\{\{x, y\},\left\{X_{i}^{y, k}, X_{i+1}^{y, k}\right\}, 0 \leq i \leq Y_{k}^{\left(T_{2}\right)}-1\right\} \stackrel{d}{=} \mathcal{F I}^{u, T_{2}} .
$$

Finally, by comparing the LHS's of (4.1) and (4.2), we get the stochastic domination in Theorem 3.1. 


\section{Asymptotic of critical values}

In this section, we prove Theorem 3.3 and Theorem 3.5. We begin the proof with inducing notations according to [9] necessary for renormalization argument.

1. Let $L_{0}$ and $l_{0}$ be positive integers to be determined later. For $n \geq 1$, let $L_{n}=l_{0}^{n} * L_{0}$ and $\mathbb{L}_{n}=L_{n} * \mathbb{Z}^{d}$.

2. Set $B_{n, x}:=x+\left(\left[0, L_{n}\right) \cap \mathbb{Z}\right)^{d}$ and $\widetilde{B}_{n, x}=\underset{y \in \mathbb{L}_{n}: d\left(B_{n, y}, B_{n, x}\right) \leq 1}{\bigcup} B_{n, y}$.

3. Let $\mathcal{I}_{n}=\{n\} \times \mathbb{L}_{n}$. For any $(n, x) \in \mathcal{I}_{n}$, write

$$
\begin{gathered}
\mathcal{H}_{1}(n, x)=\left\{(n-1, y) \in \mathcal{I}_{n-1}: B_{n-1, y} \subset B_{n, x}, B_{n-1, y} \cap \partial B_{n, x} \neq \emptyset\right\}, \\
\mathcal{H}_{2}(n, x)=\left\{(n-1, y) \in \mathcal{I}_{n-1}: B_{n-1, y} \cap\left\{z \in \mathbb{Z}^{d}: d\left(z, B_{n, x}\right)=\left\lfloor\frac{L_{n}}{2}\right\rfloor\right\} \neq \emptyset\right\} .
\end{gathered}
$$

Recall that $d(\cdot, \cdot)$ is the metric on $\mathbb{Z}^{d}$ (see Section 2 for precise definition).

4. For $x \in \mathbb{L}^{d}, n \geq 0$, let

$$
\begin{array}{r}
\Lambda_{n, x}=\left\{\mathcal{T} \subset \bigcup_{k=0}^{n} \mathcal{I}_{k}: \mathcal{T} \cap \mathcal{I}_{n}=(n, x) \text { and } \forall(k, y) \in \mathcal{T} \cap \mathcal{I}_{k}, 0<k \leq n,\right. \text { has two } \\
\text { descendants }\left(k-1, y_{i}(k, y)\right) \in \mathcal{H}_{i}(k, y), i=1,2 \text { such that } \\
\left.\mathcal{T} \cap \mathcal{I}_{k-1}=\bigcup_{(k, y) \in \mathcal{T} \cap \mathcal{I}_{k-1}}\left\{\left(k-1, y_{1}(k, y)\right),\left(k-1, y_{2}(k, y)\right)\right\}\right\} .
\end{array}
$$

By (2.8) of [9], one has

$$
\left|\Lambda_{n, x}\right| \leq\left(c_{0}(d) * l_{0}^{2(d-1)}\right)^{2^{n}} .
$$

Based on the settings above, we can decompose events of interests and estimate their probabilities by choosing proper values of $L_{0}$ and $l_{0}$. Roughly speaking, we need to select $L_{0}$ to control the 0 -level event and select $l_{0}$ to guarantee the "almost independence" between trajectories in different boxes, according to $u$ and $T$. See Figure 1 for an illustration of this renormalization scheme:

Now we give the proof of Theorem 3.3.

\subsection{Proof of Theorem 3.3}

Recall that in Theorem 3.(iii) of [3] and on Page 263 of [7], it has been shown that

$$
\limsup _{u \rightarrow \infty} u * T_{c}^{+} \leq \frac{-\log \left(1-p_{d}^{c}\right)}{2} .
$$

Thus it is now sufficient to prove that: for any $\epsilon>0$, there exists $U^{\prime}(d, \epsilon)>0$ such that for any $u>U^{\prime}$ and $T>0$ satisfying

$$
u * T \leq \frac{-\log \left(1-p_{d}^{c}\right)}{2}-\epsilon,
$$

$\mathcal{F I}^{u, T}$ does not percolate. I.e.,

$$
\liminf _{u \rightarrow \infty} u * T_{c}^{-} \geq \frac{-\log \left(1-p_{d}^{c}\right)}{2} .
$$




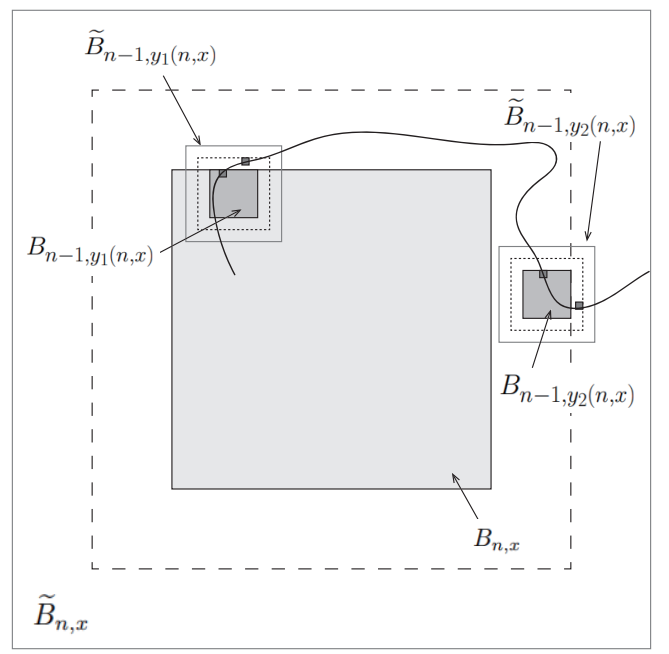

Figure 1: An illustration of the renormalization scheme

Assume that $u$ is sufficiently large while $T$ satisfies (5.4). Take $L_{0}=\left\lfloor u^{\frac{1}{2 d}}\right\rfloor>10$ and $l_{0}=10$. Let $B_{0,0}^{*}=\left\{y \in \mathbb{Z}^{d}: d\left(\{y\}, \widetilde{B}_{0,0}\right) \leq 1\right\}$ and recall the notation $\hat{\eta}^{K}$ from Section 2. By (5.4), we have

$$
\begin{aligned}
& P^{u, T}\left(\exists \eta \in \mathcal{F I}^{u, T} \text { such that }\{\eta(i), \eta(i+1)\} \subset \widetilde{B}_{0,0} \text { for some } i \geq 1\right) \\
\leq & P^{u, T}\left(\exists \eta \in \mathcal{F I}^{u, T} \text { such that the length of } \hat{\eta}^{B_{0,0}} \geq 2\right) \\
= & 1-\exp \left(-u * c a p^{(T)}\left(B_{0,0}^{*}\right) *\left(\frac{T}{T+1}\right)^{2}\right) \\
\leq & u * \operatorname{cap}^{(T)}\left(B_{0,0}^{*}\right) *\left(\frac{T}{T+1}\right)^{2} \\
\leq & c * u^{1.5} * T^{2} \leq c^{\prime} * u^{-0.5} .
\end{aligned}
$$

Let $\hat{\mathcal{F}} \mathcal{I}^{u, T}:=\left\{e \in \mathbb{L}^{d}: e=\{\eta(0), \eta(1)\}, \eta \in \mathcal{F} \mathcal{I}^{u, T}\right\}$. By Definition 2.2, one may see that $\hat{\mathcal{F}} \mathcal{I}^{u, T}$ has the same distribution as the collection of open edges of a Bernoulli bond percolation with parmeter $1-\exp \left(-\frac{u T}{(T+1)^{2}}\right)<p_{d}^{c}$.

By Theorem 6.1 of [5], we have

$$
P^{u, T}\left(B_{0,0} \stackrel{\hat{F}^{u, T}}{\longleftrightarrow} \partial \widetilde{B}_{0,0}\right) \leq\left(2 L_{0}+1\right)^{d} * P^{u, T}\left(0 \stackrel{\hat{F}^{u, T}}{\longrightarrow} \partial B_{0,0}\right) \leq\left(2 L_{0}+1\right)^{d} * e^{-c L_{0}}
$$

Note that when the event in the LHS of (5.6) does not occur, $\left\{B_{0,0} \stackrel{\mathcal{F I}^{u, T}}{\longrightarrow} \partial \widetilde{B}_{0,0}\right\}$ is equivalent to $\left\{B_{0,0} \stackrel{\hat{\mathcal{F}} \mathcal{I}^{u, T}}{\longrightarrow} \partial \widetilde{B}_{0,0}\right\}$. By (5.6) and (5.7),

$$
P^{u, T}\left(B_{0,0} \stackrel{\mathcal{F \mathcal { I }}^{u, T}}{\longrightarrow} \partial \widetilde{B}_{0,0}\right) \leq c^{\prime} * u^{-0.5}+\left(2 L_{0}+1\right)^{d} * e^{-c L_{0}} .
$$


For any $n \geq 0$ and $x \in \mathbb{L}_{n}$, we write that

$$
A_{n, x}:=\left\{B_{n, x} \stackrel{\mathcal{F I}^{u, T}}{\longleftrightarrow} \partial \widetilde{B}_{n, x}\right\} .
$$

Recall the notation $\Lambda_{n, x}$ in (5.1). For any $\mathcal{T} \in \Lambda_{n, x}$, like Equation (2.13) of [9], we write

$$
A_{\mathcal{T}}:=\bigcap_{(0, y) \in \mathcal{T} \cap \mathcal{I}_{0}} A_{0, y}
$$

Similar to (2.14) of [9], we have

$$
A_{n, x} \subset \bigcup_{\mathcal{T} \in \Lambda_{n, x}} A_{\mathcal{T}}
$$

Here we need a decoupling inequality, which is parallel to Lemma 5.4, [2]. Let $\hat{B}_{n, x}=\left\{y: d\left(\{y\}, \widetilde{B}_{n, x}\right) \leq L_{n+1}\right\}$ and $F_{n, x}:=\bigcap_{\eta \in \mathcal{F} \mathcal{I}^{u, T}, \eta(0) \in\left(\hat{B}_{n, x}\right)^{c}}\left\{\eta \cap \widetilde{B}_{n, x}=\emptyset\right\}$.

Given event $F_{n, x}^{c}$, there must exist a path $\eta \in \mathcal{F} \mathcal{I}^{u, T}$ such that $\eta \cap \partial \hat{B}_{n, x} \neq \emptyset$ and $\eta \cap \partial \widetilde{B}_{n, x} \neq \emptyset$. Recalling the construction of FRI on a finite set in Section 2 (here we take $K=\partial \hat{B}_{n, x} \cup \partial \widetilde{B}_{n, x}$ ), the number of such paths is a Poisson random variable with parameter

$$
\begin{aligned}
& u *\left[\sum_{y \in \partial \hat{B}_{n, x}} E s_{K}^{(T)}(y) * P_{y}^{(T)}\left(H_{\widetilde{B}_{n, x}}<\infty\right)+\sum_{y \in \partial \widetilde{B}_{n, x}} E s_{K}^{(T)}(y) * P_{y}^{(T)}\left(H_{\partial \hat{B}_{n, x}}<\infty\right)\right] \\
& \leq c * u *\left(L_{n+1}\right)^{d} *\left(1-\frac{1}{T+1}\right)^{L_{n+1}} \leq c_{1} u^{1.5} 10^{n d} e^{-c_{2} L_{n+1} .} .
\end{aligned}
$$

Recalling that $l_{0}=10$ and (5.12), when $u$ is sufficiently large, we have: for any $n \geq 0$,

$$
P^{u, T}\left(F_{n, x}\right) \geq \exp \left(-c_{1} u^{1.5} 10^{n d} e^{-c_{2} L_{n+1}}\right) \geq 1-e^{-c_{3} * L_{0} * 2^{n}} .
$$

Suppose that $\mathcal{T} \cap \mathcal{I}_{n-1}=\left\{\left(n-1, y_{1}\right),\left(n-1, y_{2}\right)\right\}$, where $y_{1}, y_{2} \in \mathbb{L}_{n-1}$. Since $L_{0}>10$, $\hat{B}_{n-1, y_{1}} \cap \hat{B}_{n-1, y_{2}}=\emptyset$. We denote that $\mathcal{T}_{i}=\left\{(m, y) \in \mathcal{T}: y \in \widetilde{B}_{n-1, y_{i}}\right\}, i \in\{1,2\}$, and that

$$
A_{\mathcal{T}_{i}}=\bigcap_{(0, y) \in \mathcal{T}_{i} \cap \mathcal{I}_{0}} A_{0, y} \text { and } \hat{A}_{\mathcal{T}_{i}}=\bigcap_{(0, y) \in \mathcal{T}_{i} \cap \mathcal{I}_{0}}\left\{B_{0, y} \stackrel{\left\{\eta \in \mathcal{F} \mathcal{I}^{u, T}: \eta(0) \in \hat{B}_{n-1, y_{i}}\right\}}{\longleftrightarrow} \partial \widetilde{B}_{0, y}\right\} \text {. }
$$

Note that $\hat{A}_{\mathcal{T}_{1}}$ and $\hat{A}_{\mathcal{T}_{2}}$ are independent and for $i \in\{1,2\}, \hat{A}_{\mathcal{T}_{i}} \subset A_{\mathcal{T}_{i}}$. In addition, if $F_{n-1, y_{1}}$ and $F_{n-1, y_{2}}$ both happen, the events $\hat{A}_{\mathcal{T}}$ and $\hat{A}_{\mathcal{T}_{1}} \cap \hat{A}_{\mathcal{T}_{2}}$ will then be equivalent.

By induction and (5.13), and similar to (5.26) of [2], we have

$$
\begin{aligned}
P^{u, T}\left(A_{\mathcal{T}}\right)+2 e^{-c_{3} * L_{0} * 2^{n}} & \leq P^{u, T}\left(A_{\mathcal{T}} \cap F_{n-1, y_{1}} \cap F_{n-1, y_{2}}\right)+4 e^{-c_{3} * L_{0} * 2^{n}} \\
& \leq P^{u, T}\left(\hat{A}_{\mathcal{T}_{1}} \cap \hat{A}_{\mathcal{T}_{2}}\right)+4 e^{-c_{3} * L_{0} * 2^{n}} \\
& \leq\left(P^{u, T}\left(A_{\mathcal{T}_{1}}\right)+2 e^{-c_{3} * L_{0} * 2^{n-1}}\right) *\left(P^{u, T}\left(A_{\mathcal{T}_{2}}\right)+2 e^{-c_{3} * L_{0} * 2^{n-1}}\right) \\
& \leq \ldots \leq\left(P^{u, T}\left(A_{0,0}\right)+2 e^{-c_{3} * L_{0}}\right)^{2^{n}}
\end{aligned}
$$

Combining (5.2), (5.8), (5.11) and (5.15),

$$
P^{u, T}\left(A_{n, 0}\right) \leq\left(c_{0} * l_{0}^{2(d-1)}\right)^{2^{n}} *\left(c^{\prime} * u^{-0.5}+\left(2 L_{0}+1\right)^{d} * e^{-c L_{0}}+2 e^{-c_{3} * L_{0}}\right)^{2^{n}} .
$$


Since $L_{0}=\left\lfloor u^{\frac{1}{2 d}}\right\rfloor$ and $l_{0}=10$, the sufficiently large $u$ will also guarantee that

$$
c_{0} * l_{0}^{2(d-1)} *\left(c^{\prime} * u^{-0.5}+\left(2 L_{0}+1\right)^{d} * e^{-c L_{0}}+2 e^{-c_{3} * L_{0}}\right)<0.5 .
$$

Therefore, as $n \rightarrow \infty, P^{u, T}\left(A_{n, 0}\right)$ converges to 0 and so does $P^{u, T}\left(0 \stackrel{\mathcal{F} \mathcal{I}^{u, T}}{\longleftrightarrow} \partial \widetilde{B}_{n, 0}\right)$ since $P^{u, T}\left(0 \stackrel{\mathcal{F} \mathcal{I}^{u, T}}{\longrightarrow} \partial \widetilde{B}_{n, 0}\right) \leq P^{u, T}\left(A_{n, 0}\right)$. So $\mathcal{F I}^{u, T}$ fails to percolate.

In conclusion,

$$
\frac{-\log \left(1-p_{d}^{c}\right)}{2} \leq \liminf _{u \rightarrow \infty} u * T_{c}^{-} \leq \limsup _{u \rightarrow \infty} u * T_{c}^{+} \leq \frac{-\log \left(1-p_{d}^{c}\right)}{2} .
$$

By (5.18) and Corollary 3.2, we conclude the proof of Theorem 3.3

\subsection{Proof of Theorem 3.5}

Before we give the proof of Theorem 3.5, we need an estimate on the diameter of the range of geometrically killed random walks.

Lemma 5.1. Let $\left\{X_{n}^{(T)}\right\}$ be a geometrically killed random walk with law $P_{0}^{(T)}$. Then there exist $c_{1}, c_{2}>0$ such that for all $L>0$,

$$
P_{0}^{(T)}\left[\max _{0 \leq i \leq \infty}\left|X_{i}^{(T)}\right| \geq L\right] \leq c_{1} e^{-c_{2} * T^{-\frac{1}{3}} * L^{\frac{2}{3}}} .
$$

Proof. By Theorem 1.5.1 of [6], for any positive integer $m$, we have

$$
\begin{aligned}
P_{0}^{(T)}\left[\max _{0 \leq i \leq \infty}\left|X_{i}^{(T)}\right| \geq L\right] & \leq\left(1-\frac{1}{T+1}\right)^{m}+P_{0}\left[\max _{0 \leq i \leq m}\left|X_{i}\right| \geq L\right] \\
& \leq \exp \left(-c * T^{-1} m\right)+c^{\prime} * \exp \left(-\frac{L}{\sqrt{m}}\right) .
\end{aligned}
$$

Taking $m=\left\lfloor(T L)^{\frac{2}{3}}\right\rfloor$ in (5.20) finishes the proof.

By Theorem 3.1, for any fixed $u>0, \mathcal{F I}^{u, T}$ is stochastically increasing on $T \in(0,1]$. Thus, it's sufficient to show that for $d \geq 3$ and $\epsilon>0$, there exist $\bar{c}(d, \epsilon)>0$ and $U^{\prime \prime}(d, \epsilon)>0$ such that for any $u<U^{\prime \prime}$ and $T \geq 1$ satisfying

$$
u \leq \bar{c}\left(T *(\log (T+1))^{3+\epsilon}\right)^{-(d-1)},
$$

$\mathcal{F I}^{u, T}$ does not percolate. In fact, the result proved here is sightly stronger than the statement of Theorem 3.5 and the RHS of (5.21) can be replaced by a polynomial of $T$ to make the proof a little shorter.

We use the same approach as in the proof of Theorem 3.3 but with different choices of $L_{0}$ and $l_{0}$. To be precise, we set $L_{0}=10, l_{0}=\left\lfloor\left(c^{\prime} T *(\log (T+1))^{3+\epsilon}\right)^{0.5}\right\rfloor$, where $c^{\prime}$ is to be determined later. Note that the number of paths intersecting $B_{0,0}$ in $\mathcal{F} \mathcal{I}^{u, T}$ is a Poisson random variable with parameter $u * \operatorname{cap}^{(T)}\left(B_{0,0}\right)$. Therefore,

$$
\begin{aligned}
P^{u, T}\left(0 \stackrel{\mathcal{F I}^{u, T}}{\longrightarrow} \partial B_{0,0}\right) & \left.\leq P^{u, T} \text { (there exists at least one path intersecting } B_{0,0}\right) \\
& \leq 1-\exp \left(-u * \operatorname{cap}^{(T)}\left(B_{0,0}\right)\right) \leq c^{\prime \prime} * u .
\end{aligned}
$$

For any $k \in \mathbb{N}^{+}, 0 \leq r<L_{n+1}$ and $L=k * L_{n+1}+r$, by Lemma 5.1, we have

$$
P_{0}^{(T)}\left(\max _{0 \leq i \leq \infty}\left|X_{i}^{(T)}\right| \geq L\right) \leq c_{1} \exp \left(-c_{2}^{\prime}(d) k^{\frac{2}{3}}\left(c^{\prime}\right)^{\frac{1}{3}(n+1)} T^{\frac{1}{3} n}(\log (T+1))^{\frac{1}{3}(3+\epsilon)(n+1)}\right) .
$$


Recall the notation $F_{n, x}$ in the proof of Theorem 3.3. By (5.23), when the constant $c^{\prime}(d, \epsilon)$ is sufficiently large, for $u>0$ and $T \geq 1$ satisfying (5.21),

$$
\begin{aligned}
P^{u, T}\left(\left(F_{n, x}\right)^{c}\right) \leq & \sum_{y \in\left(\hat{B}_{n, 0}\right)^{c}} P^{u, T}\left(\text { there is no path starting from } y \text { and intersecting } \widetilde{B}_{n, x}\right) \\
\leq & \sum_{L \geq L_{n+1}} c * L^{d-1} *\left[1-\exp \left(-\frac{2 d u}{T+1} * P_{0}^{(T)}\left(\max _{0 \leq i \leq \infty}\left|X_{i}^{(T)}\right| \geq L\right)\right)\right] \\
\leq & \sum_{k=1}^{\infty} c\left[(k+1) L_{n+1}\right]^{d-1} * \frac{2 d u}{T+1} * L_{n+1} \\
& * \exp \left(-c_{2}^{\prime} k^{\frac{2}{3}}\left(c^{\prime}\right)^{\frac{1}{3}(n+1)} T^{\frac{1}{3} n}(\log (T+1))^{\frac{1}{3}(3+\epsilon)(n+1)}\right) \\
\leq & c * u *\left(L_{n+1}\right)^{d} * \exp \left(-c_{2}^{\prime \prime}(d) *\left(c^{\prime}\right)^{n+1} * T^{\frac{1}{3} n} *(\log (T+1))^{\frac{1}{3}(3+\epsilon)(n+1)}\right) \\
\leq & \exp \left(-c_{2}^{\prime \prime \prime}(d) * c^{\prime} *(\log (T+1))^{\frac{1}{3}(3+\epsilon)} * 2^{n}\right) .
\end{aligned}
$$

Similar to (5.16), by (5.22) and (5.24), we have

$$
P^{u, T}\left(0 \stackrel{\mathcal{F I}^{u, T}}{\longrightarrow} \partial \widetilde{B}_{n, 0}\right) \leq\left[c_{0} l_{0}^{2(d-1)} *\left(2 \exp \left(-c_{2}^{\prime \prime \prime} * c^{\prime} *(\log (T+1))^{\frac{1}{3}(3+\epsilon)}\right)+c^{\prime \prime} * u\right)\right]^{2^{n}} .
$$

Taking $\bar{c}=\left(4 c_{0} c^{\prime \prime}\right)^{-1}$ and $c^{\prime}(d, \epsilon)>0$ large enough such that for any $T \geq 1$,

$$
c_{0} l_{0}^{2(d-1)} * 2 \exp \left(-c_{2}^{\prime \prime \prime} * c^{\prime} *(\log (T+1))^{\frac{1}{3}(3+\epsilon)}\right)<0.25 .
$$

Therefore, when $u$ is sufficiently small and $T \geq 1$ satisfies (5.21),

$$
\lim _{n \rightarrow \infty} P^{u, T}\left(0 \stackrel{\mathcal{F} \mathcal{I}^{u, T}}{\longleftarrow} \partial \widetilde{B}_{n, 0}\right)=0
$$

and thus $\mathcal{F I}^{u, T}$ does not percolate.

\section{References}

[1] L. Bowen. Finitary random interlacements and the gaboriau-lyons problem. Geometric and Functional Analysis, 29(3):659-689, 2019. MR-3962876

[2] Z. Cai, X. Han, J. Ye, and Y. Zhang. On chemical distance and local uniqueness of a sufficiently supercritical finitary random interlacement. arXiv preprint arXiv:2009.04044, 2020.

[3] Z. Cai, Y. Xiong, and Y. Zhang. On (non-)monotonicity and phase diagram of finitary random interlacement. Entropy, 23(1):69, 2021. MR-4224437

[4] J. Černỳ and S. Popov. On the internal distance in the interlacement set. Electronic Journal of Probability, 17, 2012. MR-2915665

[5] G.R. Grimmett. Percolation. Grundlehren der mathematischen Wissenschaften. Springer Berlin Heidelberg, 2013. MR-1707339

[6] G.F. Lawler. Intersections of random walks. Springer Science \& Business Media, 2013. MR-2985195

[7] A. Prévost. Percolation for the Gaussian free field and random interlacements via the cable system. PhD thesis, Universität zu Köln, 2020.

[8] E.B. Procaccia, J. Ye, and Y. Zhang. Percolation for the finitary random interlacements. arXiv preprint arXiv:1908.01954, 2019. MR-4198877

[9] P.F. Rodriguez and A.S. Sznitman. Phase transition and level-set percolation for the gaussian free field. Communications in Mathematical Physics, 320(2):571-601, 2013. MR-3053773 
Rigorous results on the phase transition of FRI

[10] A.S. Sznitman. Vacant set of random interlacements and percolation. Annals of mathematics, pages 2039-2087, 2010. MR-2680403

[11] A.S. Sznitman. Decoupling inequalities and interlacement percolation on $G \times \mathbb{Z}$. Inventiones mathematicae, 187(3):645-706, 2012. MR-2891880

Acknowledgments. The authors would like to thank an anonymous referee for helpful comments. The authors would like to thank Drs. Xinyi Li, Eviatar B. Procaccia, and Ron Rosenthal for fruitful discussions. The authors would also like to thank Dr. Eviatar B. Procaccia for allowing us to use his LaTeX macros. 


\section{Electronic Journal of Probability Electronic Communications in Probability}

\section{Advantages of publishing in EJP-ECP}

- Very high standards

- Free for authors, free for readers

- Quick publication (no backlog)

- Secure publication $\left(\mathrm{LOCKSS}^{1}\right)$

- Easy interface (EJMS²)

\section{Economical model of EJP-ECP}

- Non profit, sponsored by $\mathrm{IMS}^{3}, \mathrm{BS}^{4}$, ProjectEuclid ${ }^{5}$

- Purely electronic

\section{Help keep the journal free and vigorous}

- Donate to the IMS open access fund ${ }^{6}$ (click here to donate!)

- Submit your best articles to EJP-ECP

- Choose EJP-ECP over for-profit journals

\footnotetext{
${ }^{1}$ LOCKSS: Lots of Copies Keep Stuff Safe http://www. lockss.org/

${ }^{2}$ EJMS: Electronic Journal Management System http://www.vtex.lt/en/ejms.html

${ }^{3}$ IMS: Institute of Mathematical Statistics http://www.imstat.org/

${ }^{4}$ BS: Bernoulli Society http://www. bernoulli-society.org/

${ }^{5}$ Project Euclid: https://projecteuclid.org/

${ }^{6}$ IMS Open Access Fund: http://www.imstat.org/publications/open.htm
} 